\title{
La mise au pilori de l'abbé Maury : imaginaire comique et mythe de l'antihéros pendant la Révolution française
}

Ouzi Elyada

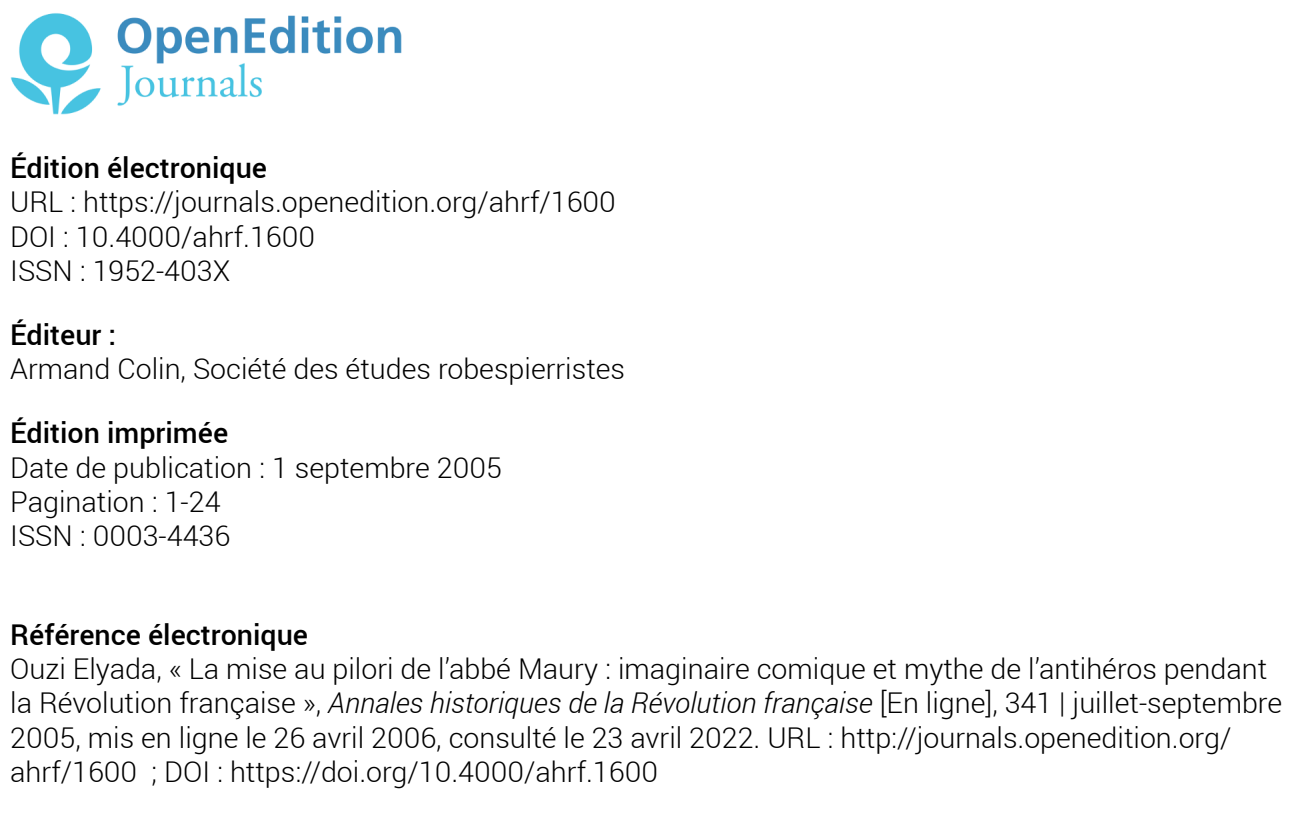

Ce document a été généré automatiquement le 23 avril 2022.

Tous droits réservés 


\title{
La mise au pilori de l'abbé Maury : imaginaire comique et mythe de l'antihéros pendant la Révolution française
}

\author{
Ouzi Elyada
}

Au centre de cette étude se trouve la question de l'usage politique de l'imaginaire pendant la Révolution française. Le phénomène imaginaire est lié à la notion de la culture politique. Pour Keith Baker, la culture politique est une série de discours, d'images et de représentations que les acteurs sociaux, individuels ou collectifs, se donnent d'eux-mêmes et de leurs adversaires et qui participent à la formation de l'identité collective et à l'attribution de sens à l'activité politique et sociale. Roger Chartier, en poursuivant la même démarche, considère le domaine de la lutte des représentations symboliques comme aussi décisive que les luttes économiques et sociales. Il constate que ce mode d'activité n'est pas réductible aux luttes sociales ; «[ ...] en développant des stratégies propres, en mobilisant des instruments et des ressources spécifiques, la représentation constitue une modalité originale et fondamentale des conflits politiques et sociaux [...]». Ces propos sont déjà fondés sur une série de travaux comme ceux de Lynn Hunt qui montre l'importance d'un personnage symbolique comme Hercule pour la compréhension de la culture politique sous la Révolution ${ }^{1}$. Mais, à côté des personnages mythologiques, on signale également l'usage médiatique de personnages réels comme Louis XVI, Marie-Antoinette ou La Fayette. Ces personnages sont représentés à travers le pamphlet, le journal, l'estampe et la chanson, dans les situations imaginaires et par rapport aux personnages fictifs (rencontre imaginaire entre le roi et Arlequin, ou visite de la reine aux enfers). Par conséquence, ces personnages bien réels se transforment en personnages imaginaires munis d'un pouvoir symbolique. Ces représentations imaginaires sont employées par les différents groupes politiques pour former l'opinion publique et lui construire une nouvelle vision du monde et une nouvelle définition de soi. 
2 Parmi les personnages symboliques les plus employés pendant les premières années de la Révolution figure l'abbé Jean Siffrein Maury. Ce député de la droite à l'Assemblée nationale, devient, à travers les pamphlets et les journaux de la gauche, un personnage imaginaire incarnant l'ennemi mythologique contre-révolutionnaire. La particularité de ce symbole réside dans son apparition dans les textes burlesques et grotesques. Pendant un an et demi, ce personnage devient la cible de nombreuses attaques parodiques qui fonctionnent comme une sorte de vengeance populaire. À travers les publications périodiques et pamphlétaires dédiées exclusivement à cette personne, on arrive à dégager le profil d'un ennemi et un mécanisme de punition collective qui sert également la cristallisation d'identité collective pour le lecteur révolutionnaire et notamment pour celui appartenant au milieu populaire parisien.

3 Dans cette étude, nous allons examiner la nature et l'usage politique de la représentation imaginaire de l'abbé Maury, dans les pamphlets et les séries périodiques entre 1789 et 1791.

1. L'abbé Maury face à Camille Desmoulins : janvier- février 1790

4 L'abbé Jean Siffrein Maury (1746-1817), fils d'un cordonnier de Valréas (comtat Venaissin), fit une brillante carrière avant la Révolution comme abbé de cour. Académicien en 1785, Maury fut élu député du clergé pour le bailliage de Péronne aux états généraux. Dans l'Assemblée constituante, il devient rapidement l'un des orateurs qui parle le plus souvent. Ce personnage pompeux, véhément, plus occupé du plaisir d'humilier ses adversaires que du désir de les vaincre, défendait avec énergie les prérogatives du clergé et de la monarchie. Il frappe l'imagination par l'érudition, le raisonnement et le nombre de ses discours. Il lui faut dire son mot sur tous les sujets. Il répète toujours le même message centré sur le passé, sur l'autorité et sur la hiérarchie sociale. Maury est doué d'une puissance vocale, d'une faculté d'improvisation et d'ironie mordante comparable à celle de Mirabeau qu'il affronte dans de célèbres débats. Porte-parole des «Noirs» royalistes, il défend avec acharnement les biens du clergé, le veto absolu et la primauté du catholicisme ${ }^{2}$.

5 Pour mieux réfuter les arguments de Maury, la presse patriotique choisit de lui répondre par un discours comique visant à ridiculiser et humilier le député de la droite. Le premier à essayer de dévaloriser le personnage de Maury fut Camille Desmoulins dans son journal, les Révolutions de France et de Brabant. Déjà à la fin de décembre 1789, dans son numéro 5, il ridiculise l'intervention de Maury à l'Assemblée nationale pendant le débat du 19 décembre 1789, où fut discutée la question des biens de l'Église :

6 " [...] Maury, voyant que le pathétique ne réussissait pas, a tiré son confrère (l'abbé de Montesquieu) par le basque et pris le ton grave, sentencieux : "Je proteste...", On a ri, "Je proteste au nom d'une province...", Il parlait de la province de Péronne, où il a été si heureux, qu'il n'y a eu point de lanterne ; on a ri à gorge déployée [...] ${ }^{3}$ ».

7 À côté de la description ridiculisant, Desmoulins insère aussi de micro-récits et des petites anecdotes comiques, visant à montrer le vrai visage du député de la droite :

8 «Le Curé De ... membre de l'Assemblée nationale, se promenait sur la terrasse de Versailles. Maury était sur les talons. L'honnête curé gémissait sur l'inacurabilié de son confrère [...]. À cette exclamation, Maury s'avance rouge de colère : "Monsieur l'abbé, sachez que j'ai toujours deux pistolets sur moi, et si..." - Si tu dis encore un mot, reprend le curé, je te donne un soufflet... L'abbé aux pistolets crut qu'il était indigne de 
lui de se battre à l'armes si inégales et l'Abbé au soufflet resta maître du champ de bataille $»^{4}$.

9 Desmoulins va continuer les attaques comiques contre Maury dans les numéros suivants. À la fin janvier 1790, dans son numéro 8, il représente une confrontation burlesque entre Maury et le secrétaire de l'Assemblée nationale. Le secrétaire avait inscrit Maury le treizième pour parler, mais Maury jurait qu'il était le troisième. Il le menaçait avec ses célèbres pistolets, mais le secrétaire n'était pas intimidé :

" Non, J.F. Maury, je ne puis faire un si grand tort à la lanterne ; vous savez bien que vous ne puissiez manquer d'être pendu d'une manière ou d'une autre: vous voudriez bien être tué par un coup de pistolet; mais je ne puis vous rendre ce service sans avoir une affaire avec M. Samson... Si vous avez tant d'envie d'être jeté ce matin dans les flammes éternelles et de rendre à Satan votre vilain âme, tuez-vous vous-même...» ${ }^{5}$.

Enfin, au début février 1790, Desmoulins ridiculise l'amour de Maury et de ses confrères pour l'argent :

«Les calotins étaient-ils si coupables d'avoir épousé de l'argent et huit cents fermes, comme l'abbé Maury... Convenons que si on lui en avait ôté une seule chaque fois qu'il a dit une sottisse dans la tribune, et qu'il s'y est rendu criminel de lèze-nation, la confiscation [de biens de l'Église] serait aujourd'hui totale ${ }^{6}$.

Face à l'abbé Maury qui domine la scène politique de l'Assemblée nationale, Desmoulins crée donc un espace alternatif, imaginaire, où cet ennemi est vaincu et humilié par les représentants de la Nation. Cette série de victoires imaginaires arrive à son apogée vers la mi-janvier 1790, à travers une gravure imagée insérée à la tête de $\mathrm{n}^{\circ} 7 \mathrm{du}$ journal. La gravure représente l'abbé Maury agenouillé devant le bureau du président de l'Assemblée nationale, lequel brandit un papier portant ces mots: «Décret contre l'abbé Maury» ; derrière Maury, un homme du peuple, debout avec un bonnet de coton sur la tête, tient à la main une lanière de cuir (un tire-pied de cordonnier) avec laquelle il s'apprête à fustiger l'abbé prosterné. Au bas de l'image, on voit l'inscription : « Punition de J.F. Maury et courroux de son père, infâme aristocrate... tu renies le tiers état " ${ }^{7}$. Cette gravure comique provoque un rire qui fonctionne par rapport au lecteur spectateur à la fois comme moyen thérapeutique et persuasif.

La vignette est conçue d'abord pour provoquer un rire négatif de mépris et de vengeance à l'égard de l'ennemi puni. Face à l'ennemi qui montre sa supériorité rhétorique dans l'Assemblée nationale, Desmoulins mobilise l'imaginaire comique pour créer des situations fictives montrant l'ennemi vaincu. Les scènes telles qu'on les montre dans la gravure sont conçues pour provoquer un rire libérateur qui situe le public dans une position de supériorité par laquelle il exprime sa joie et son soulagement en voyant l'ennemi affaibli et humilié. Cette gravure fonctionne aussi comme moyen persuasif. Du point de vue de l'auteur, les images comiques ont pour objectif de former des modes de jugement et de comportement chez le lecteur. Le rire permet à l'auteur de faciliter la communication avec le public visé, de créer un rapport de familiarité et de confiance en transformant la réception en une sorte de divertissement. En même temps, à travers l'acte de "faire rire», on pratique une technique de persuasion affective qui vise à faire croire. Le rire est aussi un moyen d'activation car en permettant la décharge émotionnelle et en créant le sentiment de libération des contraintes, il ouvre déjà la porte aux actions non contrôlées et non maitrisées par la raison. Dans la gravure, on présente un mode de comportement (Maury fouetté par un homme du peuple à l'aide d'un tire-pied), qui peut fonctionner 
comme un modèle à suivre. Mais l'auteur se sert aussi du rire pour transmettre au lecteur/auditeur populaire un message plus profond sur son identité dans le nouvel espace révolutionnaire. La situation comique est créée à partir de la notion de renversement. Maury qui, d'habitude, domine sur la scène devient dominé, battu par la Nation unie, composée de l'élite parlementaire (le président de l'Assemblée nationale) et par le petit peuple qui devient l'exécutant des décrets de l'Assemblée ${ }^{8}$.

2. Les publications périodiques 《医Maury㡠》 : février - octobre 1790

Les numéros de Camille Desmoulins de janvier - février 1790 constituent la base du Maury imaginaire. Dans les mois suivants, l'ensemble de la presse satirique, puis la presse burlesque populaire, développeront des thèmes crées par Desmoulins. La période carnavalesque qui commence fin février 1790 stimule la production de pamphlets et de journaux dédiés exclusivement à l'abbé Maury. Dans l'ensemble, j'ai trouvé six publications périodiques et une vingtaine de pamphlets publiés pendant le carnaval de $1790^{9}$.

Au début de mars 1790, on trouve à Paris une publication intitulée Les souliers de l'abbé Maury. Les deux numéros de cette série sont rédigés sous forme de récit fantastique. L'auteur joue sur le fait que le père de Maury était cordonnier dans le Comtat. À partir de ce thème, le premier numéro développe l'histoire fantaisiste de Maury arrivant à Paris portant de simples souliers, fabriqués par son père. Les souliers désignent la tendresse paternelle et l'humilité chrétienne. Le fait qu'il les a remplacés par les pantoufles cardinales lui fait oublier ses origines simples. Le symbole des souliers devient le centre d'un long discours cynique sur l'humilité ${ }^{10}$. Un deuxième périodique, publié également au début de mars 1790, portait le titre Testament de J.F. Maury. Au début du récit, Maury annonce que tout est perdu pour la contre-révolution car toutes les conspirations ont échoué. Désespéré, il se prépare à quitter ce monde cruel et il lègue à ses complices de la droite ses objets précieux : ses pistolets, ses bouteilles de vin, etc. Il lègue aussi à une fille publique du Palais Royal, une grosse somme d'argent: "[...] pour l'indemniser du dîner que je confesse lui avoir escroqué ». Puis aux rédacteurs de la presse de la droite, il lègue des rames de papier, pour qu'ils continuent à publier des discours aristocratiques. Dans le deuxième numéro, l'auteur anonyme continue à développer le thème du testament, mais cette fois dans un discours organisé en forme de récit fantastique ${ }^{11}$.

Une troisième publication «Maury», publiée vers la mi-mars 1790, porte le titre Vision et réception de l'abbé Maury. Cette publication contenant deux numéros, joue également sur le thème de la mort de l'abbé Maury (réception de l'antihéros en enfer), devenu en quelque sorte le roi du carnaval. Comme les publications précédentes, l'identité de l'auteur et de l'imprimeur demeure inconnue. Dans le premier numéro, rédigé en forme de récit fantastique, l'auteur raconte que lorsque Maury se sentit devenir l'objet du mépris et des exécrations des bons patriotes, il prit le parti d'aller trouver un refuge en Enfer. L'auteur raconte comment Maury arrive à la porte de l'Enfer et ses difficultés à y entrer car il porte un costume patriotique. Il est obligé de reprendre son habit noir. On lui permet d'entrer et on lui dit qu'il sera accueilli par tous les cardinaux, archevêques, évêques et curés, avec le pape à leur tête. Il y trouve tous les gens qui ont trahi la Révolution, volé et pillé le peuple, mais aussi des mauvais rois, des tyrans, des ministres, des magistrats, des financiers, de riches spéculateurs et des usuriers. Le numéro se termine avec Maury se voyant déjà participer aux orgies de l'Enfer. Dans le 
deuxième numéro, on décrit sa conversation avec les serviteurs du diable, au sujet de la Révolution. Par la suite, Maury est invité à prendre place à côté de Pluton ${ }^{12}$.

Vers la mi-avril, on constate que la ligne séparant l'imaginaire et le réel commence à disparaître, suite à un incident arrivé à l'Assemblée nationale. C'est en effet vers le 13 avril 1790, qu'on discute à l'Assemblée la proposition émise la veille, de décréter le catholicisme religion d'État. La séance devient vite très mouvementée et les chefs de la droite qui essayent de prendre la parole ne peuvent pas se faire entendre. Seul Maury s'accroche à la tribune sans pouvoir toutefois finir son discours. À la sortie, la foule attend les députés de la droite pour les huer et les chahuter. Certains, comme Cazalès et Mirabeau l'aîné, ne peuvent quitter les abords de l'Assemblée que protégés par la garde nationale. Quant à Maury, il doit se réfugier dans une maison de la rue Sainte-Anne. Quelques jours plus tard on distribue à Paris une gravure ridiculisant Maury. On y représente la maison où Maury a dû se cacher, comme une maison de rendez-vous. La gravure représente la foule composée de gardes nationaux et de femmes qui réclament aux filles de joie l'extradition de Maury. Ce dernier, voyant que la foule s'apprête à pénétrer la maison, se déguise en soldat et prend la fuite par le toit, en menaçant la foule avec un pistolet ${ }^{13}$. L'image de Maury, qui court sur le toit d'un bordel, armé de pistolets et protégé par les filles de joie, reste dans la mémoire collective et va nourrir la presse anti-Maury dans les mois qui suivent.

19 Une nouvelle publication Maury, qui naît vers le mi-avril 1790, va mettre en valeur le rapport entre Maury et les prostituées. Dans le numéro 3, publié vers le 14 mai 1790, on relate l'histoire de la répudiation de Maury par la négresse le lendemain de son mariage, puis son départ pour l'Enfer. L'auteur décrit l'abbé Maury qui se lève tard avec sa maitresse Esther, une ancienne fille publique, qui veut l'accompagner à l'Assemblée nationale. Il se présente à l'entrée sans cocarde. On ne le laisse pas entrer. Sa maitresse se trousse jusqu'au-dessous des hanches et montre au gardien sa jarretière nationale dont Maury va se servir comme cocarde. Esther lui accole la cocarde aux applaudissements des Suisses et des sentinelles. Ensuite, elle monte à la tribune, où elle annonce à tout le monde qu'elle est la femme de Maury. Elle intervient également à l'Assemblée, demandant de laisser parler son mari. Mais un voisin d'Esther lui explique que Maury est un ennemi du peuple, un scélérat, sans foi, sans loi, sans religion et qui va aussi répudier sa maîtresse. Convaincue des mauvaises intentions de Maury, elle rentre chez elle, trouve l'écrivain public du coin et lui dicte un acte de divorce ${ }^{14}$.

20 À la fin du temps carnavalesque, les auteurs de la presse Maury réagissent à l'activité antirévolutionnaire de l'abbé et de ses amis, à l'Assemblée nationale, par l'amplification de la violence symbolique. À l'aide de la représentation d'un monde à l'envers, ils infligent sur Maury un nombre grandissant d'humiliations et de punitions. Les scénarios de la punition ne cessent pas de varier et de s'amplifier, à travers des publications périodiques qui sont de plus en plus présentes dans l'espace public.

3. J. R. Hébert et les publications Maury

21 La plupart des rédacteurs des journaux et des pamphlets Maury en 1790 sont inconnus. En effet le seul auteur dont nous connaissons l'identité est Jean René Hébert, le futur auteur du Père Duchesne. À partir de fin février 1790, il publie une série de textes burlesques intitulés Petit carême de l'abbé Maury ou sermons prêchés dans l'assemblée des enragés ${ }^{15}$. Le sous-titre annonce le Cours complet de morale aristocratique à l'usage des jeunes gentilshommes de ce siècle. Il s'agit d'une publication contenant dix numéros publiés entre le 27 février et le 14 avril. Ils sont imprimés par Laurence Junior, libraire- 
imprimeur au $n^{\circ} 37$ de la rue Saint-Jacques. Les sept premiers numéros sont distribués d'abord au $\mathrm{n}^{\circ} 4$ de la rue Gît-le-Cœur, à l'hôtel Saint-Louis, tandis que les trois derniers le sont au $n^{\circ} 9$ de la rue Saint-Jacques, demeure de l'éditeur.

Pour Hébert, comme pour son libraire-imprimeur, la production de ce périodique est d'abord une affaire commerciale. Hébert, sans ressources depuis qu'il a été renvoyé du théâtre des Variétés amusantes au printemps 1789, se lance dans la carrière de pamphlétaire au mois de décembre de la même année. La réussite de ces premières publications rédigées également en style comique le pousse à lancer son Petit Carême ${ }^{16}$. Les textes sont rédigés en forme de sermons burlesques énoncés à la première personne par Maury devant une assemblée d'aristocrates. À la fin du premier sermon, dédié à la question des causes de la chute des grands, il donne une leçon inaugurale aux aristocrates. Maury qualifie ses auditeurs comme ceux «[...] dont la naissance supplée aux mœurs, dont le pouvoir autorise l'étendue de la volonté et des désirs et qui n'ont pas besoin de morale pour s'élever au-dessus du vulgaire, comme le cèdre s'élève audessus de l'humble arbrisseau ». Ensuite, il leur assure que "[leur] corruption personnelle n'est point un mal et que la perte de l'innocence est nulle pour ceux qui sont fait pour en imposer par leur rang au reste de la terre... vous êtes nés dans le sein des grandeurs et de la fortune que d'autres par conséquent supportent le poids du jour; que d'autres gémissent des douleurs et des calamités humaines, qu'ils succombent sous un travail pénible et continuel, qu'ils mangent un pain noir acheté à la sueur de leur front et pétri de larmes ». Par contre, pour les aristocrates : «Les plaisirs doivent entourer votre enfance et votre jeunesse, la volupté doit accompagner votre vie, la sensualité doit vous préparer une vieillesse délicieuse ${ }^{17}$.

Pourquoi donc la chute des grands? Maury l'explique par une certaine faiblesse morale :

25 " Vous rapprochez de vos esclaves par vos plaisirs, vous avez permis à ces hommes de boue de vous regarder de face; vous les avez admis dans vos cercles, vous avez souri en les voyant se glisser dans vos divines orgies et ces êtres indignes à dénouer les cordons de vos souliers, sont devenus les compagnons de vos plaisirs et de vos débauches aimables. Ils ont enfin partagé avec vous la coupe de la volupté; ces ingrats » ${ }^{18}$.

Ce genre de discours satirique se poursuit dans les numéros suivants. On y prêche contre les idées d'égalité devant la loi, de liberté et de tolérance et on demande d'établir des lois qui «n'adoucissent point le sort de l'homme qu'elle a destiné à des jours de larmes et à des nuits laborieuses, comme elle se plut à vous faire naître pour le plaisir et le bonheur. Vouloir alléger pour le peuple le fardeau de la vie, ce serait annoncer le dessin de changer les œuvres de Dieu », et le texte souhaite que le pouvoir exécutif devienne un pouvoir arbitraire et indépendant de la loi et que la dictature soit établie ${ }^{19}$. Dans le cinquième sermon publié le jour de la mi-Carême, le 2 mars 1790, on trouve face à Maury un député patriote, l'abbé Fauchet. À travers la confrontation entre le sermon patriotique et le sermon aristocratique, l'auteur ridiculise et humilie le député de la droite ${ }^{20}$. L'abbé Fauchet réapparait dans le septième numéro publié pour le jour des Rameaux ${ }^{21}$.

27 À l'aide de cette forme comique, Hébert dessine le portrait d'un ennemi de la Révolution. Tous les stéréotypes anti-aristocratiques et anti-cléricaux se trouvent dans cette publication qui couvre la période du temps carnavalesque. Le principe directeur est de créer une situation imaginaire à partir du principe de renversement où les 
intentions cachées sont dévoilées. L'apparence devient transparence. Mettre Maury dans une assemblée d'aristocrates où il peut prêcher sans crainte et dire ouvertement ce qu'il pense, à l'opposé de ses discours hypocrites à l'Assemblée ou à l'église. À l'aide du principe du renversement, le «non dit» (la parole cachée et camouflée) devient transparent, public, exposé à tout le monde. L'effet risible est amplifié grâce à la mise en scène de Maury comme sujet d'énonciation. Il est démasqué aussi à travers son discours, sa frustration face à la réussite des patriotes. Ce discours fait rire et met en colère à la fois. Il présente à la première personne le regard aristocratique sur le peuple révolutionnaire, mais ce regard est celui d'une force battue, frustrée.

Dans l'ensemble le but principal du procédé est de déclencher un rire négatif de supériorité chez le lecteur. Pourtant Maury n'est qu'un instrument, et un symbole de l'aristocratie avec ses vices, son hypocrisie, sa vision du monde arrogante, hiérarchique, et ses perversions sexuelles. En même temps, l'auteur tente de déclencher un rire libérateur face à l'ennemi dont les plans et le vrai caractère sont dévoilés. Cette transparence constitue une sorte de punition pour Maury et pour les aristocrates.

En principe, les publications ridiculisant un ennemi devraient disparaitre à la fin du temps carnavalesque, autrement dit, vers la mi-avril 1790. Pourtant, ce genre de publication représentait une bonne affaire commerciale. En conséquence, la production continua. Plusieurs entrepreneurs profitent de la fin de la série d'Hébert et inondent l'espace parisien à partir de la deuxième moitié d'avril 1790 de nombreuses publications. L'un d'eux annonce Le combat au bois de Boulogne entre Maury et Mirabeau ${ }^{22}$. On trouve également un Dialogue entre Maury et Malouet, racontant les détails d'une conspiration faite par Maury et le vicomte de Mirabeau contre la Nation, ou le miracle de l'abbé Maury et la résurrection du vicomte de Mirabeau ${ }^{23}$. Pourtant, Hébert continue à dominer le marché avec deux numéros publiés au début du mois de mai et au début de juin 1790 et intitulés Vie privée de l'abbé Maury. Hébert collabore maintenant avec un nouvel imprimeur, J. Grand, au n ${ }^{\circ} 6$ de la rue Saint-Jacques ${ }^{24}$. Dans cette publication contenant deux brochures d'une vingtaine de pages, Hébert se sert de la biographie comique, racontant la vie imaginaire de l'antihéros. Notons que dans les Annonces de la bibliographie moderne, une publication périodique qui donnait des comptes rendus sur les livres et les pamphlets révolutionnaires, on trouve, dans le numéro du 15 mai 1790, une remarque assez défavorable à l'égard de la première partie de la Vie privée : «Cette vie privée est assez mal faite en général. L'auteur y rapporte des faits qui doivent blesser des oreilles chastes $"{ }^{25}$.

En effet, cette brochure est un petit chef-d'œuvre de la littérature parodique. Hébert y montre son grand talent de conteur qui arrive à émerveiller le lecteur tout en le faisant rire. Dans la première partie du récit, Maury est ridiculisé à travers une brillante parodie de la vie de saints. Hébert amène son lecteur au petit village natal de Maury, où on voit son père, le cordelier Crespin Maury et sa mère Jacqueline la Pie医; «au bout de quatorze ans de mariage, impatient de jouir de douceurs de maternité, [cette dernière] instruit son époux des vœux secrets qu'elle avait fait d'aller en pèlerinage ». La naissance de Maury y est décrite comme le résultat d'un miracle, d'une intervention divine qui explique le fait que Maury soit devenu « la colonne de l'Église, le flambeau de sa patrie et l'admiration de son siècle ${ }^{26}$. Il semble que ce numéro ait eu un grand succès car Hébert est sollicité par son imprimeur pour rédiger une Suite de la vie privée 
de M. l'abbé Maury, qu'il publie au début du juin 1790 et qui traite de la vie de Maury durant l'année $1790^{27}$.

31 La parution des publications Maury continue jusqu'à la fin de l'année 1790, bien que leur nombre baisse. Parmi les publications pamphlétaires notons Le grand accident arrivé à l'abbé Maury occasionné par le tonnerre, publié en juillet 1790, et la Messe du 14 juillet 1790 célébrée par l'abbé Maury. Ce texte ridiculise l'antihéros pendant la fête de la Fédération. En septembre 1790 on trouve de nouveau une confrontation entre Maury et Mirabeau autour de la question des assignats. Cette campagne de pamphlets satiriques se poursuit jusqu'au mois de novembre $1790^{28}$.

4. L'abbé Maury face au père Duchesne : octobre 1790 - mars 1791

Les publications consacrées à la dévalorisation de Maury en 1790 visaient un public hétérogène. Elles devaient être diffusées dans un milieu composé à la fois par l'élite savante et par le petit peuple de la ville. Le prix bon marché des numéros, deux sols en moyenne, et le style burlesque et grotesque d'une grande partie de ces publications devaient encourager le milieu des artisans et du petit commerce de la ville à s'approprier ces numéros. Notons que les imprimés sont lus en général à haute voix dans l'espace public. À partir de l'automne 1790, Jean René Hébert s'allie à l'imprimeur Denis Tremblay, et décide de mieux cibler ses publications en se consacrant au public populaire. Cela impliquait une politique de vente bon marché et un style plus accessible à ce public, d'où le recours à partir de début septembre 1790 au genre Père Duchesne. Le principe de cette nouvelle technique d'écriture consistait à camoufler la véritable identité de l'auteur en le remplaçant par un personnage imaginaire qui s'adresse au public populaire à la première personne. Le personnage du père Duchesne est désigné comme le porte-parole du petit peuple de Paris. Il s'exprime dans un langage affectif, fort imagé, et semé d'expressions populaires, de jurons et d'injures. Les différents numéros sont rédigés en forme de monologues, de dialogues et de récits fantastiques. À travers cette forme discursive, le héros populaire exprime ses opinions sur l'événement révolutionnaire dans un style à la fois comique et violent ${ }^{29}$.

C'est à travers son journal Le père Duchesne qu'Hébert fait réapparaître le personnage de Maury. Mais si auparavant Maury régnait seul sur la scène, il la partage maintenant avec le père Duchesne. À travers une longue série de confrontations entre le personnage populaire imaginaire et le personnage réel devenu mythologique, Hébert tente non seulement de dessiner le portrait de l'ennemi, mais aussi de représenter l'image du petit peuple, ses rapports avec l'élite et son rôle pendant la Révolution.

La première rencontre entre les deux personnages a lieu au début du mois d'octobre 1790. Hébert rédige un numéro intitulé : Le père Duchesne en vendange ou sa rencontre avec l'abbé Maury à Suresnes. Il s'agit d'un numéro festif visant à faire rire le public populaire pendant la période des vendanges. Hébert est influencé ici par les numéros Maury rédigés en forme de récits fantastiques ayant pour sujet la vie sexuelle de Maury ${ }^{30}$. L'histoire se déroule pendant les vendanges à Suresnes. Le père Duchesne y est invité avec sa femme par son ami Broc pour aider les paysans. Dans la première scène on voit les vendangeurs travailler en chantant, mais alors qu'ils se préparaient à aller déjeuner, le père Broc arriva avec quelques messieurs et dames. "Tout à coup, nous apercevons une troupe de freluquets et des femmes bien pomponnées, les unes serpette à la main, les autres portant corbeilles, allons chevalier! allons, Mme la comtesse! Madame la présidente, allons! à l'ouvrage! mêlons-nous avec ces bonnes gens ». Mais le père Duchesne refuse de les accueillir: «nom d'un foutre! que je dis, est-ce que tous ces 
aristocrates vont déjà nous attrister ? C'est dit: je m'en vais si ces bougres-là restent avec nous" ".

C'est à ce moment que l'abbé Maury apparaît parmi les nobles et essaye de calmer le héros populaire : «C'est donc vous qui donnez des airs de parler, de raisonner sur tous les événements, de juger à tort et à travers, et à votre manière, les personnages les plus respectables ». Le héros après avoir reconnu l'antihéros annonce qu'il «faut damer le pion à ce bougre-là !». Par la suite, il met en garde tous les vendangeurs de la présence de l'ennemi et décide de le suivre de près. C'est là qu'il arrive à empêcher Maury de violer l'une de vendangeuses. Maury est injurié et battu par le héros populaire et, par la suite, est obligé de prendre la fuite. Le récit se termine avec la description de la gaieté populaire : «On ne se fait pas d'idée de la fête que ce fut parmi les vendangeurs quand j'eus foutu la chasse à ce foutu gredin! On rit, on but à rasade toute la journée en réjouissance de l'aventure et le soir, après les vendanges, on dansa, moi en menuet avec la mère Broc, et le père Broc pareillement avec ma femme, ensuite avec la jolie Rosière. Enfin on forma plus de vingt rondes et, foutre, je crus que l'air ça ira, ça ira allait déhancher toute la jeunesse qui était là ${ }^{31}$.

Dans ce premier récit, on trouve des composants qui vont réapparaître dans la plupart des récits de rencontre entre le héros et l'antihéros. Dans les numéros "Duchesne", Maury essaie toujours de commettre un délit contre la Nation. Il en est toujours empêché par le père Duchesne et le récit se termine en général par une punition comique de l'antihéros. Ces récits permettaient à l'auteur non seulement de montrer les vices aristocratiques mais aussi, à travers Duchesne, de représenter le peuple vigilant qui possède le droit et même le devoir d'intervenir directement contre l'ennemi.

Hébert revient à Maury au début de novembre 1790. À la suite d'une série d'interventions violentes de ce dernier à l'Assemblée nationale, Hébert rédige un numéro portant le titre: Fait beau cul et tu n'en auras guère, Ou l'abbé Maury fouetté par le père Duchesne ${ }^{32}$. Le numéro démontre bien l'usage du comique punitif comme moyen de réponse à l'ennemi réel. Le récit nous montre d'abord le père Duchesne et ses amis sortant de l'Assemblée nationale en colère, car ils venaient de voir «l'infâme calotin, ce satellite de l'aristocratie, cet enragé d'abbé Maury, prendre le brave abbé Royou et le foutre en bas de la tribune ". À coté de l'Assemblée, ils aperçoivent Maury. Ce dernier sort de l'Assemblée en voiture mais l'un de ses chevaux tombe et l'abbé est obligé de marcher : "Il jurait, foutre, à faire reculer un bataillon». Mais cela ne fait pas peur au père Duchesne et à ses amis, car « ce sont des bougres qui ont poil aux moustaches et qui savent bien ce que c'est que de jurer ». Ils décident de le suivre: "Au détour d'une rue, vis-à-vis de la boutique d'une fruitière, l'abbé est saisi par mes trois lurons, l'abbé crie, la fruitière accourt à sa porte, qu'est-ce que c'est? [...] L'abbé Maury à qui nous voulons foutre le fouet... C'est vous le père Duchesne ? Bravo, fessez fort, mais attendez donc, tenez, voilà un bouillon tout neuf, nous dit-elle, et la correction commence. L'abbé jurait, criait, se débattait, et moi l'exécuteur des hautes œuvres je l'exhortais à la soumission en lui disant, demande excuse à la nation, coquin, fait beau cul et tu n'en auras guère. Il fit un tel tapage que bientôt la rue fut inondée de spectateurs qui tous criaient, allons, allons l'abbé tu fais l'enfant, fais beau cul et tu n'en auras guère ${ }^{33}$.

Ce numéro a eu une suite inattendue qui montre bien comment l'imaginaire se mêle au réel et qui rappelle bien l'incident du 13 avril $1790{ }^{34}$. Le numéro examiné plus haut est publié le 8 novembre 1790. Le même jour, l'abbé Maury prend la parole à l'Assemblée 
nationale à la fin de la séance de l'après-midi. Il annonce : «En traversant la rue Jacob pour me rendre a l'Assemblée, j'ai rencontré un colporteur qui criait, en me suivant: Grande colère de l'abbé Maury qui a donné dans l'Assemblée nationale des coups de poing à un député Corse ». Et Maury, poursuit son récit: "Je n'ai rien dit au premier cri, ni au second, mais au troisième, j'ai saisi le colporteur et je l'ai conduit au district " ${ }^{35}$. D'après Maury, le colporteur a dit pour se justifier qu'il a crié le titre littéral d'un imprimé qu'on lui avait vendu. Maury demande qu'on s'assure de la personne du colporteur et il annonce qu'il va porter plainte contre les auteurs du libelle. D'après Maury : «En sortant du district, j'ai trouvé trente à quarante personnes de celles qu'on voit journellement à la porte de cette salle; elles m'ont hué et menacé du geste ». Il demande à l'Assemblée nationale de prendre des mesures pour que «ses membres ne soient pas ainsi exposés dans les rues et jusqu'à dans l'avenue de cette auguste enceinte ». Et il ajoute: «Les colporteurs se permettent des cris qui sont de véritables cris de haro, qui tendent à appeler le peuple contre les victimes qu'on lui désigne $"{ }^{36}$. Maury prétend qu'il ne mérite pas les attaques car, dit-il, « je n'ai mis le poing sous le nez de personne ; je n'ai attaqué aucun député de la Corse ; je n'ai donc point mérité d'être calomnié ». Pour Maury, il s'agit d'une campagne dangereuse de manipulation du petit peuple de Paris, visant à menacer et à faire taire les députés de la droite ${ }^{37}$.

Le journal parisien Révolution de Paris réagit à cette déclaration de Maury dans son numéro 70. On y trouve une gravure intitulée : Grand tumulte causé à l'Assemblée nationale par l'abbé Maury le 8 novembre 1790. On y voit l'abbé Maury dans la rue entouré du peuple saisissant un colporteur ${ }^{38}$. Le lendemain, 9 novembre 1790, Hébert répond à l'abbé Maury avec un numéro intitulé: Grande colère du père Duchesne contre l'abbé Maury. Là, à travers un monologue, le héros imaginaire parle de l'événement arrivé la veille: " Arrêter dans la rue un petit bougre de colporteur et pourquoi, par ce qu'il apprend au public que le père Duchesne a foutu le fouet à l'abbé Maury ». Hébert est en effet en colère car Maury ne mentionne pas le nom de Duchesne dans son adresse à l'Assemblée nationale: «tu as déguisé jusqu'au titre de ma feuille, de peur que les braves gens de l'Assemblée qui connaissent le père Duchesne et qui s'amusent de ses drôleries ne t'envoyassent promener avec tous les rogatons, ou plutôt c'est que tu avais honte d'avoir fait beau cul ${ }^{39}$.

La presse de la droite réagit aussi à cet incident. Suite aux attaques d'Hébert contre Maury un certain abbé Buée, secrétaire au chapitre de Notre-Dame publie un numéro visant à dévaloriser le personnage Duchesne et à mettre en valeur celui de Maury. À cet effet, il fait paraître un pamphlet intitulé : La mère Duchesne corrigeant son mari pour avoir dit du mal de monsieur l'abbé $M$. son confesseur. Dans ce texte rédigé sous forme de récit fantastique, le père Duchesne reçoit une dure leçon de la part de sa femme. Elle l'injurie, l'humilie et le jette hors de la maison ${ }^{40}$.

41 Les numéros de confrontations comiques entre Duchesne et Maury devaient avoir un très grand succès car, à partir de la mi-novembre 1790, une nouvelle publication Duchesne commence à diffuser les récits du même genre. Il s'agit du journal publié par les frères Dufour, libraires-imprimeurs de la rue du Vieux-Colombier, dont l'identité de l'auteur en cette période reste inconnue ${ }^{41}$. Vers le 16 novembre 1790 il publie un numéro intitulé L'abbé Maury poursuivi les culottes à la main par le père Duchesne. Ce numéro devait connaître plusieurs réimpressions et quelques contrefaçons. Parmi elles notons, L'abbé Maury surpris par le père Duchesne dans le boudoir de l'abbesse de Montmartre 
et grand combat qui s'est passé entre eux, et un numéro Maury intitulé, Mariage de M. l'abbé Maury avec l'abbesse de Montmartre ${ }^{42}$.

Dans la première confrontation entre Duchesne et Maury, le héros raconte comment il a été invité par les sœurs de l'abbaye de Montmartre pour réparer les fourneaux. Il fait peur aux sœurs à cause de ses injures. On le prend pour un diable et on essaie de l'exorciser. Puis il entend, « une voix forte et mâle qui partait d'une chambre voisine». Cette voix propose aux sœurs de crier «au voleur». Le père Duchesne se demande si c'est la voix de l'abbesse, qui le traite de voleur. Il ouvre une porte et trouve l'abbé Maury dans le lit de l'abbesse. Cette découverte est suivie d'un dialogue de confrontation. Duchesne qualifie Maury de «chien enragé de calotin, bouc infect, excrément de la nature...», et l'interpelle : "C'est donc ici que tu travailles sérieusement pour ton prochain...». Maury lui propose d'aller faire ses fourneaux et recaler ses cheminées. Il lui rappelle qu'il est un représentant de la Nation et qu'il doit avoir du respect à son égard. Mais Duchesne lui répond qu'il n'est pas plus un représentant de la Nation qu'un chat est représentant des souris. Il n'est qu'un scélérat qui cherche le malheur de la Nation. Ayant ainsi parlé, Duchesne songe à se retirer, mais l'abbé Maury le surprend par derrière et lui assène un coup de poing sur la tête. Là, la fureur saisit le père Duchesne et il lui envoie un coup de pied dans le derrière. "Ses culottes mal rangées lui échappent ", il les prend avec la main et se met à courir. Le père Duchesne le poursuit dans tous les corridors de la maison et il l'aurait haché si la garde n'était pas venue l'arracher de ses mains ${ }^{43}$.

43 Le journal Duchesne de la rue du Vieux Colombier continue à ridiculiser Maury au début décembre avec un numéro intitulé Nouveau projet de contre-révolution imaginé après souper par l'abbé Maury et découvert par le père Duchesne ${ }^{44}$. Ici, on ridiculise Maury à travers une histoire fantastique racontant la tentative de l'antihéros de voler la Constitution. Le récit se termine par la tentative de Maury de se sauver, assis sur un cochon, et son arrestation par le héros populaire. Ce numéro a dû également avoir un grand succès, car on le contrefait aussi ${ }^{45}$.

Dans l'ensemble, à travers ces pamphlets, le père Duchesne reste toujours le représentant du petit peuple possédant le pouvoir de surveiller à la fois la vie intime et privée et les activités politiques de l'ennemi, un thème qui sera mis en valeur aussi au début 1791 . Vers le 20 décembre 1790 un nouveau rédacteur est nommé à la tête de journal Le Père Duchesne de la rue du Vieux-Colombier. Il s'agit de l'abbé Jean Charles Jumel, l'aumônier de la garde nationale du district Saint-Lazare, membre du club des Jacobins ${ }^{46}$. Le conflit comique entre le père Duchesne et l'abbé Maury devient l'un des axes principaux de son journal. Déjà dans le premier numéro du 22 décembre 1790, il publie un récit fantastique intitulé L'abbé Maury demandant grâce au père Duchesne et lui découvre tous les dangers qui menacent la patrie. Comme dans les récits précédents, il raconte comment le héros populaire attrape l'abbé Maury en flagrant délit de séduction de sa fille Rosette. Maury demande grâce et révèle au père Duchesne tous les complots contre la Nation ${ }^{47}$.

45 Cette petite histoire sert de base à l'abbé Jumel pour expliquer la nature d'une vignette imagée insérée à la tête de ce numéro. Au centre de cette vignette, on trouve le père Duchesne dans son atelier entouré de fourneaux et de poêles. C'est un véritable Gargantua, un type moustachu, gros et gras, d'une quarantaine d'années portant un chapeau rond et des vêtements populaires. Il tient à sa main droite une hache et porte deux pistolets passés dans la ceinture. On aperçoit également un fusil derrière. Face à 
lui se trouve l'abbé Maury. C'est une petite personne bien coiffée et bien habillée, tremblant de peur qui se trouve agenouillée devant le héros, demandant grâce. Notons que cette image diffère de la gravure publiée vers la mi-avril 1790, où l'on voyait Maury menaçant la foule avec ses pistolets. Ici, par contre, Maury, désarmé, tremble de peur face au héros populaire qui a l'air souriant et décontracté, une pipe allumée dans sa bouche et une bouteille de vin vide à côté. Au-dessous de la vignette, on trouve l'inscription «Mémento Mori, sacrée calotte».

Cette vignette vise d'abord à déclencher chez le lecteur populaire un rire méchant de supériorité face à l'abbé Maury humilié et châtié, mais en même temps la vignette transmet une certaine vision utopique du monde désiré par le petit peuple. En effet, la vignette se réfère à la tradition carnavalesque du monde à l'envers. Car depuis deux siècles on voit se répandre en France des gravures à destination populaire distribuées notamment pendant le temps carnavalesque. Elles montrent une série de scènes comiques fondées sur le principe du renversement. On trouve des images montrant l'artisan puni par son apprenti, le mari battu par sa femme, l'enfant donnant des ordres à ses parents, ou l'instituteur châtié par son élève ${ }^{48}$. La démonstration de Duchesne qui punit Maury est plus qu'une confrontation entre un patriote et un aristocrate. Il s'agit d'une représentation de renversement entre la culture populaire et la culture des élites. C'est le petit peuple qui se présente armé, c'est lui qui gouverne et qui possède le droit de punir, face au représentant de la culture d'élite mis dans une position d'infériorité. Il semble donc que l'auteur transmette à travers cette vignette une représentation d'un pays de cocagne où le petit peuple domine seul et baigne dans le plaisir et l'abondance (vin, tabac), la sécurité (armes) et la joie.

Rappelons que onze mois plus tôt, en janvier 1790, Camille Desmoulins publia une gravure représentant le député de la droite agenouillé et demandant grâce. Pourtant la gravure de l'abbé Jumel est beaucoup plus subversive. On a vu que dans la gravure de Desmoulins, Maury est puni par un homme du peuple qui porte une lanière de cuir à la main, mais ici, la véritable autorité est détenue par le président de l'Assemblée nationale dont le petit peuple est le bras exécutant. Par contre, dans la gravure de l'abbé Jumel la scène ne se déroule pas dans l'espace officiel de l'Assemblée, mais entièrement dans l'espace populaire où le personnage populaire apparaît comme la seule incarnation du pouvoir, celui du petit peuple ${ }^{49}$.

Cette vignette imagée fonctionne d'abord pour l'abbé Jumel comme moyen de distinction par rapport à ses concurrents. En effet, pendant l'année 1791 on trouve à Paris huit publications périodiques centrées autour du père Duchesne. Ces publications sont rédigées en même style mais elles servent à la fois la gauche radicale, le centre patriotique et la droite antirévolutionnaire.

Jumel, qui soutient les Jacobins, pense que ses lecteurs pourront distinguer sa publication grâce à la vignette "mémento Mori». Mais cette vignette est vite contrefaite, d'abord par Hébert (à partir de son numéro 13 du 19 janvier 1791), et ensuite par trois autres publications «Duchesne», dont une liée à la droite antirévolutionnaire. Notons qu'Hébert va garder cette même vignette à la tête de son journal pendant plus de trois ans, jusqu'à la mi-mars $1794{ }^{50}$. Pourtant, le rédacteur du journal Duchesne de la rue du Vieux Colombier se sert également de cette vignette pour un autre objectif. Car à partir de début janvier 1791, il lance une campagne contre les prêtres refusant de prêter le serment ${ }^{51}$. Pour l'abbé Jumel, Maury est l'incarnation des prêtres réfractaires et à travers son image il essaie durant la première moitié de 1791 
de former une opinion publique populaire anti-cléricale. Déjà vers le 5 janvier 1791 , dans un numéro publié pour la fête des Rois, il publie un dialogue intitulé L'abbé Maury conduit à Bicêtre par le père Duchesne pour avoir refusé de prêter son serment civil. Comme dans les numéros précédents, on assiste ici à une mise au pilori comique de l'abbé Maury à travers une interrogation comique de l'antihéros par le père Duchesne sur les causes de son refus de prêter le serment, une interrogation terminée par l'aveu de Maury qu'il est un ennemi de la Nation ${ }^{52}$. Ce texte burlesque démontre bien l'usage du genre «Maury». L'auteur se sert d'images comiques pour représenter le petit peuple comme le vrai souverain possédant le droit d'interroger, de juger et même d'exécuter ses propres verdicts, un verdict représenté par le sommaire de ce numéro où selon la logique du renversement, le prêtre est envoyé à Bicêtre par le petit peuple. La punition comique de Maury continue deux semaines plus tard. Suite au discours de Maury à l'Assemblée nationale en faveur de prêtres réfractaires au serment, Jumel publie un numéro annonçant : L'abbé Maury chassé par le père Duchesne de la tribune de l'Assemblée à coups de tuyau de poêle pour avoir provoqué l'enfer et tous les diables contre l'instruction sur la Constitution civile du clergé ${ }^{53}$.

Hébert, à l'opposé de Jumel, utilise moins Maury comme symbole des réfractaires mais plutôt comme l'incarnation de la force anti-révolutionnaire. C'est dans ce sens qu'il réagit au discours de Maury concernant les pensionnaires des Invalides avec le numéro du 17 février 1791 intitulé: La grande joie du père Duchesne et l'abbé Maury fouetté par les vivandiers des Invalides pour avoir ajourné le décret en leur faveur ${ }^{54}$. Dans un autre récit fantastique qui date de la fin février 1791, ce sont les femmes et les forts de la Halle qui ridiculisent et humilient l'abbé Maury ${ }^{55}$.

51 Aux deux publications Duchesne traitant l'abbé Maury, s'ajoute à la fin février 1791 une troisième. Il s'agit d'un journal lié au club des Cordeliers, également dirigé par un ecclésiastique, l'abbé Robin, un ancien censeur royal. Ce journal publié à un rythme quotidien, fut imprimé par mademoiselle Colombe à la place Henri IV. Ajoutons qu'il s'agit de l'un des imprimeurs parisiens les plus radicaux, qui publie au même moment L'Ami du Peuple de Marat et L'Orateur du Peuple de Fréron ${ }^{56}$. Robin comme Hébert ne s'intéresse pas à Maury le prêtre, mais à Maury l'aristocrate. Notons que Robin comme Hébert contrefait la vignette «Mémento Mori» de Jumel et qu'il se sert même de la graphie «Mori» pour désigner ce personnage. Au début de mars 1791 Paris se prépare de nouveau pour le carnaval. Face à l'interdiction officielle de cette fête ${ }^{57}$, les publications comiques basées sur la notion du monde à l'envers se multiplient de nouveau et c'est durant cette période que l'on va voir le genre Maury atteindre son apogée, notamment à travers les trois journaux «Duchesne» de la gauche.

Pour le jour de mardi gras l'abbé Robin publie le 8 mars 1791 Le grand carnaval du père Duchesne et son grand combat avec l'abbé Maury à qui il a cassé le nez... ${ }^{58}$. L'auteur rappelle au début que le carnaval est interdit : "Que le carnaval a chez nous la mine pâle et blême! Nous avons tous l'air de fondeurs de cloches, et l'un ne rit à Paris que d'une lèvre...». Mais le héros aime bien se divertir et il est assez content de son mardi gras. Il raconte qu'il a fêté le carnaval avec le duc d'orléans. "La fête a été belle, le repas excellent. Au premier couvert, nous avons mangé tous les égouts des huit cents fermes de l'abbé Maury, au second, toutes les pommes de terre de la Normandie, mais au troisième couvert, la table se remplit de poulardes, de gélinottes et de brailles gras comme les cuisses des chamoisent. Mangez, s'écria l'hôte, la révolution le permet à tout le monde...». Mais à ces mots, un bruit se fait entendre, et on voit entrer l'abbé Maury, 
Cazalès et Foucault. Les trois sont arrivés pour manger. «Tous ces morceaux, s'écrit le requin à tonsure, nous appartiennent, ils sont faits pour nos dents. C'est nous qui les croquerons... Après ce discours, les trois mâtins portent la patte sur les plats. À la vue de cette audace, le feu monta sur tous les visages. Bougre, s'écria le bon Philippe, vous me le payerez, sacré dieu, et à l'instant il prit un potage tout bouillant et le leur jette sur les doigts. Les trois brigands échaudés, lâchèrent alors prise et se retirent dans un coin en se soufflant les doigts...» ${ }^{59}$. Mais Maury et ses amis ne sont pas découragés : "Quand la douleur fut passée, les trois chiens reviennent à la charge et voulurent nous chasser de table. Mille noms d'une crosse, dit l'abbé Maury, d'une voix de bouledogue : rendez-vous ces chapons et ces poulets, ils sont à nous, vous nous les avez volés...». C'est à ce moment que le père Duchesne décide d'intervenir: « À ce coup, je ne pus plus retenir ma colère, je me lève sur mes pieds, je saisis le mâtin de l'abbé par la gueule, je prends une pleine main de pommes de terre restées sur mon assiette, je le lui enfourne dans le gosier avec une fourchette qui y rentra aussi. Tiens, bougre, lui dis-je, tu as jusqu'à ici de poulardes, il faut que tu mange à son tour de topinambour: cependant le coquin allonge la jambe pour me foutre un coup de pied, je l'empoigne aussitôt par son sacré croupion et le lance de toutes mes forces, contre la tête de Cazalès et tombe sur le dos de Foucault» ${ }^{60}$.

Hébert ne tarde pas à publier des textes du même genre comme La grande joie du père Duchesne à l'occasion de la victoire remportée par les paysans du Comtat sur les aristocrates au camp de Jales et son grand combat entre le père de l'abbé Maury et un chanoine de Carapateras, ou La grande députation des dames et des forts de la Halle ayant à leur tête le père Duchesne pour complimenter l'abbé Maury de ce qu'il a été nommé cardinal et lui porter le chapeau rouge ${ }^{61}$. On trouve également un texte de l'abbé Jumel du même genre publié vers le 6 mars $1791^{62}$.

La grande réussite de textes Maury peut expliquer également la décision d'Hébert de reprendre la publication de son journal satirique Petit Carême de l'abbé Maury. Rappelons qu'Hébert publia la première série de 10 numéros pendant le temps carnavalesque de 1790. Maintenant, pour le temps carnavalesque de 1791, il relance la publication à l'aide de son éditeur Denis Tremblay. Pour cette deuxième série, j'ai trouvé 3 numéros, publiés entre le début et la fin mars $1791{ }^{63}$. Tous les numéros sont organisés sous forme de sermon comique. Le premier sermon pour le mercredi des cendres, montre Maury qui prêche à la chapelle du Pape en présence du père Duchesne. Les deux autres numéros contiennent des sermons prêchés par Maury pour le premier et le deuxième dimanche de Carême, l'un devant une assemblée des prêtres réfractaires, et l'autre devant le roi dans sa chapelle du Louvre. Cette deuxième série n'a pas eu un grand succès, car Hébert a dû arrêter la publication après trois semaines, sans arriver à la fin du temps carnavalesque. Il me semble qu'au printemps 1791 le texte satirique est moins demandé que le texte burlesque et grotesque, plus facile à déchiffrer et plus violent.

5. La disparition des imprimés Maury de la scène révolutionnaire

55 Les publications mettant en scène l'abbé Maury disparaissent progressivement à l'automne 1791. Cette baisse de la production des textes correspond à la fin de l'Assemblée constituante et à la prochaine réunion de l'Assemblée législative. Maury n'était pas élu à la nouvelle assemblée et en conséquence la presse populaire patriote commence déjà vers la fin août 1791 à se désintéresser du personnage. 
grande colère du père Duchesne qui a encore une fois foutu les entrevoies à l'abbé Maury avec le tire-pied d'un savetier du coin, pour le punir de tout le mal qu'il a fait à la Nation et de tous les complots qu'il médite encore ${ }^{64}$. Au début du récit le héros populaire se demande pourquoi depuis quelques jours on ne voit plus l'abbé Maury à l'Assemblée. Duchesne trouve deux raisons à cette absence : la première est qu'il est gêné de voir triompher la Nation et la Constitution terminée, malgré lui. La seconde, qu'il a eu peur en voyant expirer le temps de l'inviolabilité. En attendant la contre-révolution, Maury se promène à Paris déguisé : « Tantôt en poissarde, tantôt en dragon, tantôt en fort de la halle, il court tous les quartiers de Paris et sur tous les faubourgs ; sous ces différents costumes il se plaît à tourmenter les badauds en leurs annonçant la guerre civile, la famine, la ravage, la désolation et la peste.... ${ }^{65}$.

Dans ce récit, Maury essaie de se déguiser en père Duchesne. C'est sous ce masque qu'il est arrêté par les commères du quartier. Mais Maury n'arrive pas à convaincre les femmes: «Toi, le père Duchesne, bougre de réchappe de Bicêtre, tu crois donc que nous ne le connaissons pas? Tu ignore donc que c'est notre voisin, notre compère... nous t'allons conduire chez lui, il t'apprendra à prendre sa ressemblance...». On amène Maury à la boutique du père Duchesne où il est reconnu par le véritable héros : « Savezvous qui il est ? C'est le cardinal Savate, lui-même le jean-foutre d'abbé Maury... À ces mots, une grêle de coups de pieds et de coups de poings lui tombe sur la carcasse... ah, lui dis-je à mon tour, te voilà encore une fois sous ma main. Tu devrais avoir appris à ne te pas frotter au père Duchesne. Oh, tu vas encore danser aujourd'hui...». L'histoire se termine par la scène montrant Maury demandant grâce : "Le bougre tombe à mes pieds et les mains jointes dans la même attitude qu'on le voit dans ma gravure, il me supplie de ne pas mettre au vent son postérieur. Culottes bas, lui dis-je, sinon, foutre, j'emporte la pièce». Duchesne refuse donc de gracier Maury qui est fouetté puis est obligé de demander pardon à la Nation ${ }^{66}$.

Avec ce dernier récit, s'achève le genre Maury de période révolutionnaire. Dès lors, on ne trouvera plus le personnage du célèbre orateur de l'Assemblée constituante au centre de nouveaux textes pamphlétaires. Pourtant le personnage Maury ne va pas disparaître complètement de l'espace public. Dans les années suivantes, on va continuer à lire en public les anciens numéros de confrontation entre Maury et Duchesne. Encore en été 1793 le Journal de la Montagne signale que les lecteurs publics qui montent sur des tréteaux construits sur le Pont-au-Change, lisaient devant les passants des dialogues entre l'abbé Maury et le père Duchesne ${ }^{67}$.

Pourquoi donc, le personnage Maury a disparu de la scène pamphlétaire? Maury occupe un rôle important dans l'Assemblée constituante comme chef de file de la droite antirévolutionnaire. Ce brillant orateur de la droite qui tente sans cesse d'empêcher le travail de législation révolutionnaire, attire la presse satirique et populaire qui le considère comme le plus grand obstacle à l'achèvement de la constitution. Il est donc logique qu'avec la fin de son mandat comme député à l'Assemblée constituante, la presse patriotique, satirique et populaire, va se désintéresser de lui. Pourtant, la présence de Maury dans cette presse commence à baisser quelques mois plus tôt, vers la fin du temps carnavalesque de 1791. Il me semble que la raison principale de ce changement est liée à la radicalisation des camps patriotiques. Il est vrai que pour la presse patriote, le personnage Maury incarne parfaitement les deux grands ennemis de la Révolution: le prêtre et l'aristocrate (ou plutôt l'homme du peuple devenu aristocrate); mais au printemps 1791, ce personnage ne constitue plus un symbole 
assez puissant pour mobiliser le peuple vers une révolution plus égalitaire et plus républicaine. Pour radicaliser l'opinion populaire il fallait un symbole d'ennemi plus puissant et plus menaçant. Petit à petit, à partir du printemps 1791, la presse satirique et populaire commence à remplacer le personnage Maury, comme ennemi du peuple, par les personnages de la reine Marie-Antoinette, et du roi, Louis XVI. Comme nous l'avons montré ailleurs, la presse populaire remplace le personnage Maury par celui du roi, bien avant la fuite à Varennes. Et c'est à partir de cette période que les personnages du roi et de sa femme sont ridiculisés et punis à travers les mêmes procédés de l'imaginaire comique carnavalesque utilisés auparavant contre Maury ${ }^{68}$.

\section{NOTES}

1.Sur la définition de la notion «culture politique», voir Keith Michael Baker, Inventing the French Revolution, Cambridge, Cambridge Univ. Press, 1990, pp. 4-11 ; Roger Chartier, Les origines culturelles de la Révolution française, Paris, éditions du Seuil, 1990, pp. 167-188 ; id., Au bord de la falaise, Paris, Albin Michel, 1998, pp. 77-84, 99-102. Sur l'usage de l'imaginaire pendant la Révolution, voir Bernadette Fort, «The French Revolution and the Making of Fictions», pp. 13-32, dans Fictions of the French Revolution, sous la direction de Bernadette ForT, Northwestern Univ. Press, 1991. Voir aussi dans ce volume l'article de Jacques REVEL, « Marie Antoinette and Her Fictions, The Staging of Hatred», pp. 111-129; Lynn HUNT, Politics, Culture, and Class in the French Revolution, Berkeley, University of California, 1984, pp. 52-119. Annie Duprat, Le roi décapité : essai sur les imaginaires politiques, Paris, Cerf, 1992 ; id., Le Rois de papier : la caricature de Henri III à Louis XVI, Paris, Belin, 2002, pp. 108-147 ; Ouzi Elyada, «L'usage de personnages imaginaires dans la presse et le pamphlet pendant la Révolution française», Revue d'Histoire moderne et contemporaine, 44-3 (juillet-septembre 1997), pp. 484-503.

2.Sur Maury voir Xavier Raduget, La carrière politique de l'abbé Maury de 1786 à 1791, Paris, Letouzey, 1912 ; Louis Maury, vie du cardinal Jean Siffrein Maury, par L. S. Maury son neveu, Paris, Gayet, 1828 ; Georges Bonnet-Maury, Le cardinal Maury d'après ses mémoires et correspondances, Paris, 1892 ; Jean-François Poujoulat, Le cardinal Maury, sa vie et ses ceuvres, Paris, 1855 ; Edna Hindie Lemay, Dictionnaire des constituants, 1789-1791, Paris, Universitas, 1991, vol II, pp. 645-648.

3.Révolutions de France et de Brabant, n 5, pp. 193-194.

4.Ibid., n 5, pp. 195-196.

5.Ibid., nº 8, pp. 347-349.

6.Ibid., $\mathrm{n}^{\circ} 10$, pp. 436-437.

7.Révolutions de France et de Brabant, $\mathrm{n}^{\circ} 7$.

8.Desmoulins continue l'attaque comique contre Maury en fin janvier et au début février 1790. Voir, ibid. ${ }^{\circ} 8$, pp. 347-349, $n^{\circ} 10$, pp. 436-437.

9.Pour la liste complète des publications Maury, voir Ouzi Elyada, Presse populaire et feuilles volantes de la Révolution à Paris 1789-1792: inventaire méthodique et critique, Paris, Société des Études Robespierristes, 1991, pp. 177-190.

10.Les souliers de l'abbé Maury, BN, $8^{\circ} \mathrm{LC} 2-369,2$ numéros.

11.Testament de J.F.Maury, BN, $8^{\circ} \mathrm{LB} 39-3253-4$. 
12.Visions et réception de l'abbé Maury, $\mathrm{BN}, 8^{\circ} \mathrm{LC} 2-2323$.

13.Pour cette gravure, voir Yann Fauchois, Religion et France révolutionnaire, Paris, Herscher, 1989, p. 55.

14. L'abbé Maury, BN, $8^{\circ} \mathrm{LC} 2-2359, \mathrm{n}^{\circ} 3$. Voir aussi $\mathrm{n}^{\circ} 6$.

15.Petit carême de l'abbé Maury ou sermons prêchés dans l'assemblée des enragés, BN, $8^{\circ} \mathrm{LB} 39-3019$.

16.Sur Hébert avant 1790 voir, M. Grey, Le père Duchesne agent royaliste, Paris, Perrin, 1983.

17.Petit Carême..., op. cit, $\mathrm{n}^{\circ} 1$, pp. 1-3.

18.Ibid., p. 3-4.

19.Ibid., $\mathrm{n}^{\circ} 2$, pp. 11-13.

20.Ibid., $\mathrm{n}^{\circ} 5$.

21.Ibid., $\mathrm{n}^{\circ} 7$.

22.Détail du combat qui a eu lieu ce matin au bois de Boulogne entre M. l'abbé Maury et Mirabeau, BN, $8^{\circ}$ LB39-9538.

23.Voir, O. Elyada, «Presse populaire...», op.cit., pp. 181-182, ns 11, 18, 25.

24. Vie privée de l'abbé Maury, BN 8LB39-3256.

25. Annonces de la bibliographie moderne, tome II, $\mathrm{n}^{\circ} 60 \mathrm{du}$ 15/5/90.

26.Vie privée..., op. cit.

27. Suite de la vie privée de M. l'abbé Maury, BN, 8²B39-8657.

28. Grand accident arrivé à l'abbé Maury occasionné par le tonnerre, BN, $8^{\circ}$ LB39-865. Messe du 14 juillet 1790 célébrée par l'abbé Maury, BN, 8²B39-3770. Voir aussi, O. Elyada, op.cit, ns 3 et 8 pour le mois du septembre et $n^{\circ} \mathrm{s} 5$ et 15 pour le mois de novembre 1790 .

29.Sur le père Duchesne voir, F. Braesch, Le père Duchesne d'Hébert, Paris, 1938. Jacques Guilhaumou, «Les mille langues du père Duchêne, la parade de la culture populaire pendant la Révolution», dans Dix-huitième siècle, n¹8, 1986, pp. 145-146. id., La langue politique de la Révolution française, Paris, 1989, pp. 182-184. id. L'avènement des porte-parole de la république (1789-1792), Paris, Presses universitaire du septentrion, 1998.

30.Le père Duchesne en vendange ou sa rencontre avec l'abbé Maury à Suresnes, BN, $8^{\circ}$ LB39-2540.

31.Ibid.

32.Le Père Duchesne d'Hébert, $\mathrm{BN}, 8^{\circ} \mathrm{LC} 2-512, \mathrm{n}^{\circ} 5$. Le titre de ce numéro devait être influencé par un autre publié par Chalon, Faites beau cul, vous n'auriez qu'une claque, ou événement arrivé à l'abbé Maury dans la cour du collège Mazarin, fouetté par les écoliers, le 27 à trois heures, BN, $8^{\circ} \mathrm{LB} 39-8590$. On y raconte l'histoire de Maury rendant visite à son ami professeur au collège Mazarin, mais reconnu par les écoliers qui le font fouetter par « Painblanc», le correcteur du collège.

33. «Fait beau cul...», op. cit., pp. 4-7.

34.Le rapport entre le débat à l'Assemblée nationale le 13 avril 1790 et la gravure ridiculisant Maury, publiée par la suite.

35.Le moniteur universel, $\mathrm{n}^{\circ}$ 314, 10 novembre 1790. «Compte rendu de séance de 9 novembre», p. 329.

36.Ibid.

37.Ibid.

38. Les Révolutions de Paris, $\mathrm{n}^{\circ} 70$.

39. Le Père Duchesne d'Hébert, $\mathrm{BN}, 8^{\circ} \mathrm{LC} 2-512, \mathrm{n}^{\circ} 7$.

40.La mère Duchesne corrigeant son mari pour avoir dit du mal de monsieur l'abbé M. son confesseur, BN, $8^{\circ}$ LC2-3884. Sur ce texte voir aussi O. Elyada, «La Mère Duchesne : 
masques populaires et guerres pamphlétaires : 1789-1791», Annales historiques de la Révolution française, 1988, n 1, pp. 1-16. O. Elyada, (éd.) Lettres bougrement patriotiques de la Mère Duchesne, Paris, Les Editions de Paris/EDHIS, 1989, pp. 4-9.

41.Sur le journal de la rue du Vieux Colombier, voir, O. Elyada, «La représentation de l'image royale avant Varennes», Annales historiques de la Révolution française, 1994, n 3, pp. 529-531.

42. L'abbé Maury surpris par le père Duchesne dans le boudoir de l'abbesse de Montmartre et grand combat qui s'est passé entre eux, BN, $8^{\circ} \mathrm{LC} 2-2542$; Mariage de M. l'abbé Maury avec l'abbesse de Montmartre, BN, $8^{\circ}$ LB39-8559.

43. L'abbé Maury poursuivi les culottes à la main par le père Duchesne, BN, $8^{\circ} \mathrm{LC} 2-2444$.

44. Nouveau projet de contre-révolution imaginé après souper par l'abbé Maury et découvert par le père Duchesne, $\mathrm{BN}, 8^{\circ} \mathrm{LC} 2-2321$.

45.Voir par exemple: Départ de l'abbé Maury à cheval sur un cochon portant la constitution en croupe, arrêté par le père Duchesne, BN, $8^{\circ}$ Lc2 2322.

46.Sur Jumel, voir O. Elyada, «La représentation...», op. cit.

47. Le père Duchesne de la rue du Vieux Colombier, BN, $8^{\circ} \mathrm{LC} 2-513, \mathrm{n}^{\circ} 2$.

48.F. Tristan, Le monde à l'envers, Paris, Hachette, 1980.

49. Révolutions de France et de Brabant, $n^{\circ}$ 10, 30/1/1790.

50.Sur les contrefaçons de la vignette Mémento Mori, voir, O. Elyada, «Presse populaire...», op. cit., pp. 247-260.

51.O. Elyada, « Le rire anticlérical dans la presse populaire révolutionnaire ", dans Conflits politiques, controverses religieuses - essais d'histoire européenne aux 16e-18e, 0 .

Elyada, Jacques LE BRUN, (éd.), Paris, Editions de l'EHESS, 2002, pp. 241-257.

52.Le Père Duchesne de la rue du Vieux Colombier, BN, $8^{\circ} \mathrm{LC} 2-513$, no. 1.

53.Ibid., $\mathrm{n}^{\circ} 25$.

54.Le Père Duchesne d'Hébert, BN, $8^{\circ} \mathrm{LC} 2-508, \mathrm{n}^{\circ} 30$.

55.Ibid., $\mathrm{n}^{\circ} 37$.

56.Sur le journal Henri IV, voir F. BRAESCH, op. cit., pp.74-76. Voir aussi, O. ELYADA, Presse populaire et feuilles volantes..., op. cit., pp. 140-146.

57.Sur l'interdiction du carnaval, voir, O. Elyada, « La Presse populaire parisienne et le temps du carnaval : 1788-1791 », dans M. Vovelle (éd.), L'image de la Révolution française, Paris et Londres, Pergamon Press, 1989, V. I, pp. 108-118

58. Le Père Duchesne de la place Henri IV, BN, 8 LC2-519, $\mathrm{n}^{\circ} 17$.

59.Ibid., pp. 3-4.

60.Ibid., pp. 4-7

61.Le Père Duchesne d'Hébert, op. cit., ns 40, 37.

62.Le Père Duchesne de la rue du Vieux-Colombier, op. cit., $\mathrm{n}^{\circ} 48$.

63.Petit Carême de l'Abbe Maury ou sermons prêches dans l'assemblée des enragées deuxième série, $n^{\circ} \mathrm{s} 1-3, \mathrm{BN}, 8$ Lb 39 3019. Pour les sommaires des numéros et leur datation, voir, O. Elyada, Presse populaire et feuilles volantes..., op. cit., p. 184.

64.Le père Duchesne d'Hébert, op. cit., $\mathrm{n}^{\circ} 79$.

65.Ibid., pp. 2-3.

66.Ibid., pp. 7-8.

67.Albert Soboul, Les sans-culottes, parisiens en l'an II : mouvement populaire et gouvernement révolutionnaire 1793-1794, Paris, Publications de la Sorbonne, 1958, p. 670-1 68.O. ELYADA, «La représentation populaire de l'image royale avant Varennes», op. cit. 


\section{RÉSUMÉS}

$\mathrm{Au}$ centre de cette étude se trouve la question de la violence symbolique pendant la Révolution française. On examine ici la pratique de la violence symbolique contre l'abbé Maury à travers le discours comique de la presse satirique et de la presse populaire sous l'Assemblée constituante. L'abbé Maury, chef de la droite contre-révolutionnaire, devient la cible d'une campagne de ridiculisation à partir de l'hiver 1789. On étudie ici l'évolution des attaques comiques contre Maury à partir du journal de Camille Desmoulins les Révolutions de France et de Brabant. Celui-ci fournit entre décembre 1789 et février 1790 les grands éléments du mythe diabolisant Maury. Par la suite, on voit apparaître pendant le temps carnavalesque (février - mai 1790) un grand nombre de pamphlets et de publications périodiques satiriques consacrés exclusivement à l'abbé Maury. Mais la campagne contre Maury arrive à son apogée entre septembre 1790 et mai 1791 avec la naissance de la presse populaire Le Père Duchesne. À travers trois journaux Père Duchesne patriotiques, nous examinons ici la mise en scène d'une confrontation comique entre le père Duchesne, symbole du petit peuple et l'abbé Maury. À travers une centaine de numéros, les auteurs construisent un espace imaginaire qui sert de lieu ludique où l'on montre la punition et l'humiliation de Maury et la victoire du petit peuple. Nous montrons que les auteurs de ces scènes introduisent progressivement en 1791 la notion de la souveraineté populaire. Avec la fin de l'Assemblée constituante on voit disparaître le personnage de Maury de la scène pamphlétaire. À partir de cette période, ce sont les personnages de la reine et du roi qui deviennent la cible préférée du discours populaire burlesque et grotesque.

Pillorying priest Maury : comic portrayal and myth of the anti - hero during the French Revolution. This survey mainly deals with the issue of the symbolical violence during the French Revolution. This violence is analysed through the humorous tone of the satirical press and the popular press denouncing priest Maury at the time of the Constituent Assembly. Being the leader of the counter - revolutionary right wing, he becomes the target of a mockery campaign from Winter 1789 onwards. The evolution of these comic attacks is analysed from Camille Desmoulins's newspaper Les Révolutions de France et de Brabant. Between December 1789 and February 1790, Camille Desmoulins provides the main elements of the myth denouncing Maury. Later, during the carnival (February - May 1790 ), a great deal of lampoons and satirical periodicals are exclusively dedicated to him. But the campaign against Maury reaches its peak between September 1790 and May 1791 with the birth of the popular press and more particularly with the different versions of Le Père Duchesne. In three of them, we are presented a comic confrontation between le Père Duchesne, symbolizing people of modest means and priest Maury : in about a hundred issues, the authors present a play scene in which the first one is victorious whereas the second is punished and humiliated. The notion of popular sovereignty is progressively introduced by these authors. But, at the end of the Constituent Assembly, the character of Maury disappears from the lampoonist stage and is replaced by the characters of the Queen and the King who become the favourite targets of the comic and ludicrous popular press.

\section{INDEX}

Mots-clés : presse, violence symbolique, pamphlets, carnaval, abbé Maury, abbé J.-Ch. Jumel, J.R. Hébert 
AUTEUR

OUZI ELYADA

Université de Haifa, département d'histoire générale, Haifa, Mont Carmel, Israel 